\title{
Adenomatous Hyperplasia of Thyroid Nodule Classification: Texture Feature Analysis Methodoloyof Ultrasound Images
}

\author{
S Kohila, G. Sankara Malliga
}

\begin{abstract}
This paper, explores to extract textural feature from ultrasound Adenomatous Hyperplasia thyroid nodule. The extracted texture feature will help the clinician to improve diagnostic accuracy. The Fine Needle Aspiration (FNA) and Histopathology report is the conventional diagnostic methodology. It is an invasive technique and patients are subjected to painful process. Non-invasive, non-contact and low cost imaging tool is essential to increase the clinical diagnostic accuracy. Ultrasound imaging is a potential non-invasive modality to capture Adenomatous Hyperplasia thyroid nodule. Adenomatous Hyperplasia thyroid nodule is the common inflammation in thyroid gland abnormality. The texture features, using Law's Texture Energy Measures (Law's TEM), Neighborhood Gray Tone Difference Matrix (NGTDM) and Statistical Feature Matrix (SFM) are extracted from normal and adenomatous hyperplasia of thyroid nodules. The normal and abnormal images are demarcated using T-test analysis. The extracted feature shows significant difference between normal and abnormal images with $p$ value less than $0.001(p<0.001)$. Hence the Law's Texture Energy based feature may be used to identify the pathology in the thyroid ultrasound images.
\end{abstract}

Keywords: Adenomatous Hyperplasia, Texture analysis, Law's Texture Energy Measures, NGTDM, Statistical Feature Matrix

\section{INTRODUCTION}

The thyroid is an endocrine gland, a small, and butterflyshaped in front of the neck. It has two lobes and isthmus connects these two lobes. The thyroid hormones control the body. The abnormal overgrowths of tissue in the thyroid gland are the thyroid nodules. The nodules can be benign or malignant. Detection of thyroid abnormality at the early stage is very much essential. Ultrasound is an efficient tool to detect the thyroid nodules.

Ultrasound images with less manual intervention are helpful to radiologist in the detection and diagnosing of the nodules. An efficient algorithm to classify normal and abnormal

thyroid

nodulesareessential.Thiseliminatesthepainfulbiopsy.Adenom atoushyperplasiaofthyroidisacommon type of thyroid nodule. These nodules may turn to malignant later. This paper aims to detect the Adenomatous hyperplasia of thyroid using ultrasound imaging only. If these nodules could be detected at an early stage using only ultrasound images, there are many advantages such as preventing the nodule to turn to malignant, avoids unnecessary anesthesia, painful surgeries.

Revised Manuscript Received on 14 August, 2019.

S Kohila, Associate Professor, Department of Electronics and Communication Engineering, Sri Sairam Engineering College.Research scholar, Vels University,Chennai, Tamilnadu, India.(Email: kohila.ece@sairam.edu.in)

Dr. G. SankaraMalliga, Professor and Head, Department of Electronics and Communication Engineering, Anand Institute of higher technology, Chennai, Tamilnadu, India. (Email: sankaramalliga@gmail.com)
Several research works have been done on the thyroid ultrasound images to demarcate the nodule into normal and pathological subjects. In a study the thyroid ultrasound features are discussed and ultrasound features [1] such as, calcification, irregular margins and shape are associated with malignancy and smooth appearances associated with benign nodules. Discrete WaveletTransform is used on ultrasound imaging [2] to decompose the image, and various image features are extracted and analyzed to classify the benign nodule and malignant nodule. In a study the ultrasound features [3] to classify the malignancy as well as the recommendation for fine needle aspiration are discussed. In another research work [4], the importance of CAD systems on the classification of malignant and benign thyroid nodules are highlighted. Texture feature based classification of normal and Hashimoto's ultrasound thyroiditis are reported in [5]. Fractal analysis was attempted to differentiate normal and Hashimoto subject of thyroid ultrasound images [6]. Texture features were extracted [7] using two-threshold binary decomposition.

Many literature reported that, from the thyroid ultrasound images, features based on textural properties are efficient in classifying the normal and pathological subjects such as malignant and hashimoto's thyroid images. The extensive survey shows that there is no study related to the performance of texture analysis on adenomatous hyperplasia of thyroid. Hence adenomatous hyperplasia of thyroidis investigated from the texture feature of normal and abnormal images. This will enable the clinicians to manage the disease process, avoid the progression of abnormality, which in turn eliminates anesthesia and serious surgeries.

In this study, computer aided diagnosis is addressed to classify adenomatous hyperplasia of thyroid and normal images. In general invasive procedures such as FNAC or histopathology methods are adopted. Law's TEM , SFM textures and NGTDM features are extracted from normal and abnormal ultrasound images. The significant features are chosen based on student $\mathrm{T}$ - test analysis. The student T-test shows significant delineation between Normal and adenomatous hyperplasia of thyroid.

\section{MATERIALS AND METHODS}

Thirty five images of Adenomatous hyperplasia thyroiditis and Twenty five normal images are collected with biopsy report from a Ultrasound diagnostic Centre at Chennai, India. The diagnosis is validated using histopathology reports. Typical normal and abnormal thyroid 


\section{ADENOMATOUS HYPERPLASIA OF THYROID NODULE CLASSIFICATION: TEXTURE FEATURE ANALYSIS METHODOLOY OF ULTRASOUND IMAGES}

ultrasound images are shown in Figure 1 and Figure 2. The region of interest are cropped manually using interactive computer aided tooland shown in figure2.

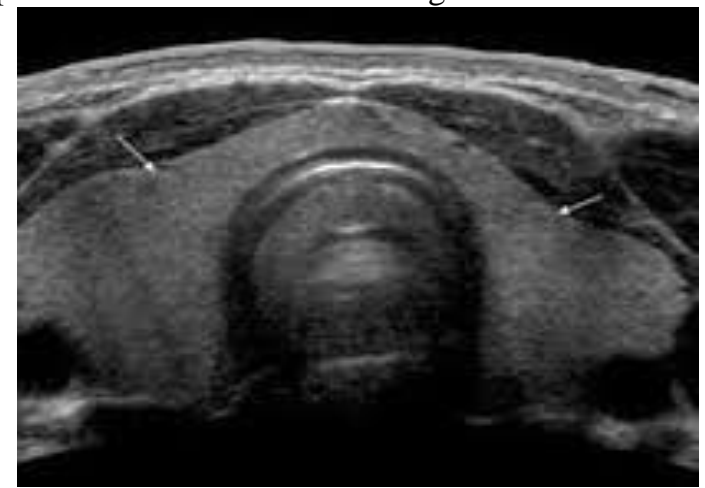

Fig. 1: Ultrasound image of thyroid (without nodule)
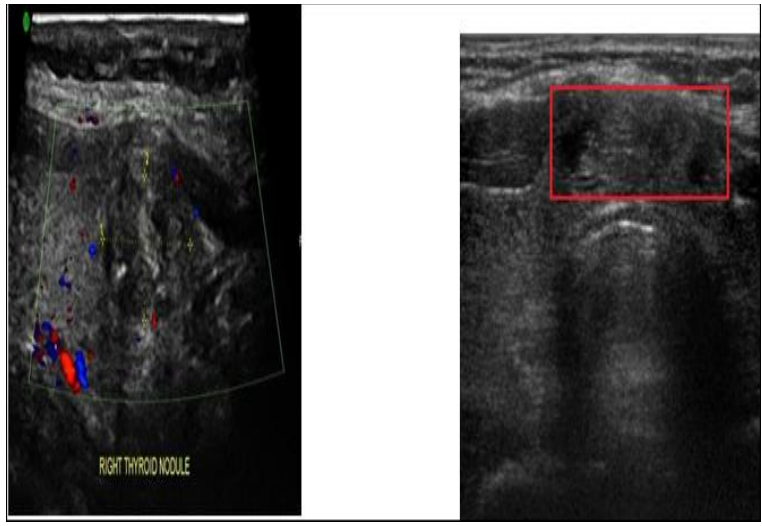

Figure 2: Ultrasound image of thyroid (with nodule marked by the Radiologist(left) and manually marked(right))

A maximum number of $N X N$ non-overlapping images are chosen to extract the texture samples. Features were extracted using Laws Texture Energy Measures methods, Neighbourhood Gray Tone Difference Matrix and Statistical Feature Matrixfrom all images and Student T- test is used to identify the significant features.

\section{NEIGHBORHOOD GRAY TONE DIFFERENCE MATRIX (NGTDM)}

Amadasunet al [8] reported coarseness, contrast, complexity, texture strength, and busyness discriminate the textural patterns in the images.

Coarseness refers to the relatively large particles and quality of pixel, which is given as,

$f_{\cos }$ :

Where Gmax is the maximum intensity of the image, and

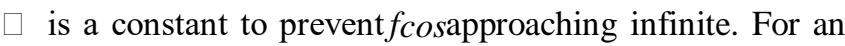
MXM image, the occurrences of gray tone value $\mathrm{i}$ in terms of probability $P i$ is given by

\section{$P i=M i / n 2 \quad$ where $n=M-2 d$.}

Dissimilarity of patterns depict the Contrast, the computational form for this property is given as,

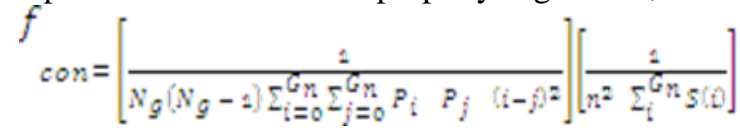

Where, $N g$ is the number of different intensity levels in the image,

$$
N_{g}=\Sigma_{i}^{G_{k}} Q_{i \text { Where, }} Q_{i}=\left\{\begin{array}{l}
1, \text { if } P_{i} \neq 0 \\
0, \text { otherwise }
\end{array}\right.
$$

The $f_{c o n}$ is a product of two terms.

The computational measure of busyness is,

$$
f_{b u s}=\sum_{i}^{G_{n}} P_{i} S(i) / \sum_{i}^{G_{n}} \sum_{i}^{G_{n}} i P_{i}-j P_{j}
$$

Where $\mathrm{Pi} \neq 0, \mathrm{Pj} \neq 0$ Complexity is given as

$$
\mathrm{f}_{\text {comp }} \sum_{i}^{C_{n}} \sum_{j}^{G_{n}}\left\{(|i-j|) /\left(n^{2}\left(P_{i}+P_{j}\right)\right)\right\}\left\{P_{i} S(i)+P_{j} S(j)\right\}
$$

where $\mathrm{Pi} \neq 0, \mathrm{Pj} \neq 0$ Strength is ,

$$
f_{\text {str }}=\left[\sum_{i}^{G_{n}} \sum_{j}^{G_{n}}\left(P_{i}+P_{j}\right)(i-j)^{2}\right] /\left[\varepsilon+\sum_{i}^{G_{n}} S(i)\right]
$$

Coarseness, Contrast, Complexity and Strength are NGTDM features extracted from the adenomatous hyperplasia of thyroid ultrasound images.

Statistical Feature Matrix (SFM)

Cheng[9], suggested Coarseness, Contrast, Periodicity, Roughness features which are common to all visual textures.

\section{LAW'S TEXTURE ENERGY MEASURES}

Texture energy measures are efficient in capturing the intensity of variation within a fixed size window[10]. Laws developed level, edge and spot vector for the feature extraction purpose. The level vector computes the local average, edge vector. It detects the edges and spot vectors to identify the spot energy in the images. These vectors are combined with each other or convolved among themselves to obtain six energy map LL, EE, SS, LE, ES and LS vectors of size $3 \mathrm{X}$ 3.The resultant vector is applied over the image and the features are calculated. These six featuresextracted from the normal and abnormal images are analyzed using studentT-test.

\section{RESULTS}

A total of 900 features ( 15 features from 35 abnormal and 25 normal images) were extracted, computed and analysed. ROI of uniform size is cropped manually for image analysis. The extracted features are statistically analysed. T - Test was performed between the adenomatous hyperplasia of thyroid and Normal groups and is presented in Table 1-3. The significant features which differentiate the normal and abnormal images are highlighted. Statistical significance of $\mathrm{p}<0.001$ are considered to be significant features. Based on the analysis the four features extracted using NGTDM and

\begin{tabular}{|c|c|c|c|c|c|}
\hline Texture features & \multicolumn{5}{|c|}{ NGTDM } \\
\hline T-Test & coarsne & contras & busyne & Complexi & Strengt \\
\hline & ss & $\mathrm{t}$ & ss & ty & $\mathrm{h}$ \\
\hline $\begin{array}{l}\text { Between Normal } \\
\text { and Adenomatous }\end{array}$ & 0.00268 & 0.0004 & 0.9470 & 0.00000 & 0.0000 \\
\hline $\begin{array}{c}\text { hyperplasia of } \\
\text { thyroid }\end{array}$ & & 0 & 1 & & 3 \\
\hline
\end{tabular}
Law's TEM features can be used to differentiate the thyroid disorders using the ultrasound images.

Table 1. T - Test Analysis using NGTDM 
Table 2. T Test Analysis usingSFM

\begin{tabular}{|c|c|c|c|c|}
\hline Texture features & \multicolumn{4}{|c|}{ SFM } \\
\hline T-Test & coarseness & contrast & periodicity & roughness \\
\hline $\begin{array}{c}\text { Between Normal } \\
\text { and Adenomatous } \\
\text { hyperplasia of thyroid }\end{array}$ & 0.00326 & $\mathbf{0 . 0 0 0 0 0}$ & $\mathbf{0 . 0 0 0 0 0}$ & $\mathbf{0 . 0 0 0 0 0}$ \\
\hline
\end{tabular}

Table 2. T Test Analysis using Law's TEM

\begin{tabular}{|c|c|c|c|c|c|c|}
\hline Texture features & \multicolumn{7}{|c|}{ Law's TEM } \\
\hline T-Test & LL & EE & SS & LE & ES & LS \\
\hline BetweenNormal & $\mathbf{0 . 0 0 0 0 0}$ & $\mathbf{0 . 0 0 0 0 0}$ & $\mathbf{0 . 0 0 0 0 0}$ & $\mathbf{0 . 0 0 0 0 0}$ & $\mathbf{0 . 0 0 0 0 0}$ & $\mathbf{0 . 0 0 0 0 0}$ \\
\hline $\begin{array}{c}\text { and Adenomatous } \\
\text { hyperplasia } \\
\text { ofthyroid }\end{array}$ & & & & & \\
\hline \\
VI. CONCLUSION & & & & & \\
\hline
\end{tabular}

The CAD system may reduce the subjective errors.This paper shows that the diseased thyroid tissue can be differentiated from normal thyroid tissue by means of image texture analysis of ultrasound images. The significant features shall be fed to classifier may give betterclassification results. The study on adenomatous hyperplasia of thyroid using ultrasonogram images may help to predict the severity at an early stage and thereby painful fine needle aspiration can beavoided.

\section{REFERENCES}

1. Remonti, L. R., Kramer, C. K., Leitao, C. B., Pinto, L. C. F., \& Gross, J. L. (2015). Thyroid ultrasound features and risk of carcinoma: a systematic review and metaanalysis of observational studies. Thyroid, 25(5), 538550.

2. Sudarshan, V. K., Mookiah, M. R. K., Acharya, U. R., Chandran, V., Molinari, F., Fujita, H., \& Ng, K. H. (2016). Application of wavelet techniques for cancer diagnosis using ultrasound images: A Review. Computers in biology and medicine, 69, 97-111.

3. Kim, E. K., Park, C. S., Chung, W. Y., Oh, K. K., Kim, D. I., Lee, J. T., \&Yoo, H. S. (2002). New sonographic criteria for recommending fine-needle aspiration biopsy of nonpalpable solid nodules of the thyroid. American Journal of Roentgenology, 178(3), 687691.

4. Acharya, U. R., Swapna, G., Sree, S. V., Molinari, F., Gupta, S., Bardales, R. H.,...\&Suri, J. S. (2014). A review on ultrasound-based thyroid cancer tissue characterization and automated classification. Technology in cancer research \& treatment, 13(4), 289301.

5. Kohila, S. and Malliga, G.S., 2016, August. Classification of the Thyroiditis based on characteristic sonographic textural features and correlated histopathology results. In Signal and Image Processing (ICSIP), IEEE International Conference on(pp. 305309). IEEE.

6. Omiotek, Z. (2017). Fractal analysis of the grey and binary images in diagnosis of Hashimoto's thyroiditis. Biocybernetics and Biomedical Engineering, 37(4), 655665.

7. Prochazka, A., Gulati, S., Holinka, S., \&Smutek, D. (2019). Patch-based classification of thyroid nodules in ultrasound images using direction independent features
8. Amadasun, M., \& King, R. (1989). Textural features corresponding to textural properties. IEEE Transactions on systems, man, andCybernetics, 19(5), 1264-1274.

9. Wu, C. M., \& Cheng, Y. C. (1992). Statistical feature matrix for texture analysis. CVGIP: Graphical Models and Image Processing, 54(5), 407-419.

10. Laws, K. I. (1979, November). Texture energy measures. In Proc. Image understanding workshop (pp. 47-51). 